Louisiana State University

LSU Digital Commons

4-1-2019

\title{
Ionic conductivity and counterion condensation in nanoconfined polycation and polyanion brushes prepared from block copolymer templates
}

\author{
Christopher G. Arges \\ Cain Department of Chemical Engineering \\ Ke Li \\ Louisiana State University \\ Le Zhang \\ Cain Department of Chemical Engineering \\ Yu Kambe \\ Pritzker School of Molecular Engineering \\ Guang Peng Wu \\ Department of Polymer Science and Engineering, Zhejiang University
}

See next page for additional authors

Follow this and additional works at: https://digitalcommons.Isu.edu/chemistry_pubs

\section{Recommended Citation}

Arges, C., Li, K., Zhang, L., Kambe, Y., Wu, G., Lwoya, B., Albert, J., Nealey, P., \& Kumar, R. (2019). Ionic conductivity and counterion condensation in nanoconfined polycation and polyanion brushes prepared from block copolymer templates. Molecular Systems Design and Engineering, 4 (2), 365-378.

https://doi.org/10.1039/c8me00081f

This Article is brought to you for free and open access by the Department of Chemistry at LSU Digital Commons. It has been accepted for inclusion in Faculty Publications by an authorized administrator of LSU Digital Commons.

For more information, please contact ir@lsu.edu. 


\section{Authors}

Christopher G. Arges, Ke Li, Le Zhang, Yu Kambe, Guang Peng Wu, Baraka Lwoya, Julie N.L. Albert, Paul F. Nealey, and Revati Kumar 


\section{Ionic conductivity and counterion condensation in nanoconfined polycation and polyanion brushes prepared from block copolymer templates}

Christopher G. Arges ${ }^{a^{*}}, K_{e}$ Li $^{b}$, Le Zhang ${ }^{a}, Y u K_{\text {Kambe }}^{c, d}$, Guang-Peng Wu $u^{e}$ Baraka Lwoya ${ }^{f}$, Julie N. L. Albert ${ }^{f}$ Paul F. Nealey ${ }^{c, d^{*}}$, and Revati Kumar ${ }^{b^{*}}$

${ }^{\mathrm{a} C a i n}$ Department of Chemical Engineering, Louisiana State University, Baton Rouge, LA 70803

${ }^{\mathrm{b}}$ Department of Chemistry, Louisiana State University, Baton Rouge, LA 70803

${ }^{\mathrm{c}}$ Institute for Molecular Engineering, University of Chicago, Chicago, IL 60637

${ }^{\mathrm{d}}$ Materials Science Division, Argonne National Laboratory, Argonne, IL 60439

${ }^{\mathrm{e}}$ MOE Key Laboratory of Macromolecular Synthesis and Functionalization, and Key Laboratory of Adsorption and Separation Materials \& Technologies of Zhejiang Province, Department of Polymer Science \& Engineering, Zhejiang University, Hangzhou 310027, China

${ }^{\mathrm{f}}$ Department of Chemical and Biomolecular Engineering, Tulane University, New Orleans, LA 70118

*Corresponding authors: carges@1su.edu; revatik@1su.edu; nealey@uchicago.edu

\section{ABSTRACT}

Microphase separated block copolymer electrolyte (BCE) systems are attractive candidates for electrochemical systems because the concentrated ionic groups in one block provide pathways for facile ion transport while the non-ionic block bestows mechanical integrity and suppresses excess water uptake. Numerous researchers have prepared and studied bulk BCEs as ion conductors, but these materials inherent complex nature and imprecise architecture makes it difficult to extract definitive conclusions how the macromolecular structure influences ion transport. In this work, the process of block copolymer lithography was demonstrated to create model nano-confined polymer electrolyte brushes that mimic lamellae structures found in bulk BCE membranes. Both nano-confined anion and cation conducting polymer brushes were prepared and excellent ionic conductivities $\left(10^{-2} \mathrm{~S} \mathrm{~cm}^{-1}\right.$ to $\left.10^{-1} \mathrm{~S} \mathrm{~cm}^{-1}\right)$ were obtained. 
Surprisingly, the nano-confined polycation and polyanion brushes displayed similar, or in some instances lower, in-plane electrical resistance values to the non-confined samples and shrinkage upon introduction of ionic charges. Using 2D force mapping AFM and atomistic molecular dynamics simulations, it was inferred that the nano-confined polymer electrolytes were less susceptible to counterion condensation explaining the unexpected changes in brush thickness after introduction of ionic moieties and the relatively low electrical resistance values. Overall, the block copolymer lithography platform presented in this work enables fabrication of precisely defined BCEs to systematically investigate how microstructure and confinement governs counterion condensation and ion transport.

\section{DESIGN, SYSTEM, and APPLICATION}

Nanostructure attributes are critical factors that govern ionic conductivity in polymer electrolytes. In this work, block copolymer lithography is shown as a powerful platform to fabricate nanostructured channels of polycation and polyanion brushes into predefined patterns. Using a multi-faceted approach of molecular dynamics simulations, 2D atomic force mapping, and impedance spectroscopy, it is demonstrated that nanoconfinement of polymer electrolytes reduces counterion condensation and enhances ionic transport rates. The implications of these results with model systems are useful for advancing solid-state electrolytes for a broad array of electrochemical technologies that include fuel cells, batteries, and ionic separations.

\section{INTRODUCTION}

Ion-conducting polymer electrolytes are central components to several types of electrochemical devices that include batteries, fuel cells, sensors, electrolyzers, ionic separations, 
and water purification units. ${ }^{1-6}$ The polymer electrolytes provide electron insulation and ionic conduction while often also serving as a mechanical separator between the two electrodes. Thin polymer electrolyte variants, e.g., $1 \mu \mathrm{m}$ in thickness or less, do not have the mechanical integrity to serve as a bulk separator for sandwich type electrochemical cells. However, they have found recent application to planar, nascent technologies such as nanoionics ${ }^{7,8}$, which are targeted for non-volatile memory, and thermal galvanics for waste heat recovery ${ }^{9,10}$. Furthermore, studying microphase separated polymer electrolytes in the thin film format has yielded insights to how the microstructure of these materials influences ionic conductivity. ${ }^{11-15}$

Thin film polymer electrolyte ionic conductivity studies on interdigitated electrode (IDE) setups ${ }^{11,15}$ with a targeted morphology are akin to studying Faradaic reaction kinetics in thin film, well-defined electrocatalyst structures with a rotating disk electrode setup. ${ }^{16,17}$ This approach for electrocatalyst characterization is commonly performed before deploying the catalyst materials into electrolyzers and fuel cell devices because the complex nature of bulk 3D electrodes obfuscates attainment of the intrinsic reaction rate coefficients. Hence, studying thin film ionic conductivity of block copolymer electrolytes (BCEs) on IDEs, similar to investigations of Faradaic reactions in thin film electrocatalyst structures, is useful because they display the same microstructures observed in bulk, 3D membranes but with a greater degree of fidelity and precision of the microstructure. The value proposition of thin film studies lies in the greater confidence in correlating microstructure to ionic conductivity as structural defects within the thin film can be annihilated ${ }^{18,19}$ or significantly reduced.

Maximizing the ionic conductivity of polymer electrolytes is an important priority for electrochemical devices as it can lower the ohmic overpotential resulting in greater thermodynamic efficiency. A common strategy to improve conductivity is to increase the ionic 
loading of the polymer electrolyte, but this strategy is not without its flaws. Continued increase in ionic loading comes with the consequence of poor mechanical properties and excessive swelling. ${ }^{20,21}$ The desired properties for polymer electrolytes for electrochemical applications is high ionic conductivity and mechanical resiliency, but these two distinct attributes are often inversely related when increasing the ionic loading within these materials. ${ }^{22-24}$

Microphase separated BCEs ${ }^{11,23-36}$ offers a compromise to the mechanical integrity-ionic conductivity tradeoff because concentration of the ionic groups yields high ionic conductivity with lower ionic loadings and the non-ionic block domain endows the material with better mechanical properties. Block copolymers can be systematically manipulated into various microstructure morphologies at the nano level (e.g., cylinders, bi-continuous/gyroid, lamellae, and spheres with periodic feature sizes of 3 to $50 \mathrm{~nm}) .{ }^{28,37} \mathrm{In}$ our recent perspective ${ }^{22}$, we highlighted some of the shortcomings of BCE current studies that relate microstructure to material ionic conductivity. The literature survey inspired us to develop systems and tools that definitively correlate structural factors of BCEs (e.g., morphology, grain boundaries, and aligned domains) to bulk ionic conductivity. To address the knowledge gap, our groups have leveraged block copolymer lithography ${ }^{38}$ and directed self-assembly ${ }^{39}$ to make ordered BCE structures that allow one to connect the salient features of the microstructure to ionic conduction.

In this report, nanoconfined polymer electrolyte brushes, which resemble microphase separated lamellae structures in bulk membranes, were prepared using block copolymer template assisted lithography. ${ }^{38,40}$ Both single ion-conducting polycation and polyanion variants were prepared and their lamellae structures were confirmed via scanning electron microscopy (SEM) and atomic force microscopy (AFM). Thin film materials, as reported in our previous works ${ }^{11,15}$, were useful for understanding how terminal defect sites and percolation dictate ion transport in 
BCEs. However, our previous method was limited to anion conductors. The samples prepared in this work investigate both anion and cation conductors and demonstrate bulk conductivity values up to $10^{-2}$ to $10^{-1} \mathrm{~S} \mathrm{~cm}^{-1}$ at $20^{\circ} \mathrm{C}$ in deionized water. Upon analyzing our results, we observed that nanoconfined polymer electrolyte brushes had similar or lower resistance values to planar substrates covered completely with polymer electrolyte brushes. Additionally, the planar substrates with the polymer electrolyte brushes displayed a reduction in film thickness upon introduction of ionic groups. These observations hinted that nanoconfined polymer electrolyte brushes might display less counterion condensation ${ }^{41-48}$ than substrates covered completely with polymer electrolyte brushes. The condensed counterions are posited for resisting transport in the presence of an electrochemical potential gradient. As a result of the initial conductivity and film

thickness findings, 2D force microscopy ${ }^{49,50}$ and atomistic molecular simulations were deployed to further substantiate the presence of counterion condensation in the nano-confined and nonconfined polymer electrolyte brushes. The approach disseminated here enables future possibilities to: i.) systematically investigate how long-range ordered structures (e.g., domains with a tortuosity of one or with precisely defined 90 degree bends or jogs ${ }^{38,51}$ ) influence ionic conduction and ii.) to see how counterion condensation, under confinement in polymer electrolyte brushes, impacts ionic transport.

\section{RESULTS AND DISCUSSION}

Figure 1a depicts the fabrication scheme used to prepare nano-confined sulfonated poly(styrene) $\left(\mathrm{H}^{+}\right.$counterion; nc-sPS $)$and nano-confined poly(2-vinyl n-methyl pyridinium iodide) (nc-mP2VP) polymer electrolyte brushes from block copolymer templates. The samples with no ionic groups were designated nc-PS and nc-P2VP. The nano-confined polymer 
electrolyte brushes were prepared by first performing self-assembly of poly(styrene-blockmethyl methacrylate) (PS- $b$-PMMA $\mathrm{Mn}_{\mathrm{M}: 38 k-36.8 k}$ ) on a non-preferential crosslinked mat composed of poly(styrene-random-methyl methacrylate-random-glycidyl methacrylate) (PS-r-PMMA-rPG $-76 \mathrm{wt} \%$ styrene and $4 \mathrm{wt} \%$ glycidal methacrylate $)^{52-54}$. After spincoating PS- $b$-PMMA on the non-preferential layer, thermal annealing under nitrogen environment generated fingerprint lamellae structures with a natural periodic spacing $\left(L_{\mathrm{o}}\right)$ of $37 \mathrm{~nm} .{ }^{55}$ Subsequently, UV radiation ( $\lambda=254 \mathrm{~nm}, 40 \mathrm{~mJ} \mathrm{~cm}^{-2}$ dose) degraded the PMMA block in the self-assembled BCP and glacial acetic acid wet-etched the degraded polymer block. Upon removing the PMMA block, the uncovered underlying crosslinkable mat was dry-etched using oxygen reactive ion etching. It is important to note that the methyl methacrylate units in the PS-r-PMMA-r-PG may have also been degraded by the wet-etch process, but their relatively small weight fraction (20\%) and the crosslinks from the glycidal methacrylate kept the crosslinked mat layer intact. $1 \mathrm{wt} \%$ of monohydroxy terminated polymer brush (either mono-hydroxyl terminated polystyrene (OH-PS) or mono-hydroxy terminated poly(2-vinyl pyridine) (OH-P2VP)) in toluene was spincoated on top the nanopatterned crosslinked mat. The mono-hydroxy terminated functionalized polymers were thermally grafted to the exposed SiOx in the nanoconfined regions. Afterwards, unreacted brush and the PS domain from the etched BCP were rinsed away by immersion in excess toluene ${ }^{56}$ under sonication. Subsequent chemical reactions introduced ionic moieties into the grafted brush regions. For nc-sPS samples, the nc-PS brushes were exposed to acetyl sulfonate solution to tether sulfonic acid moieties to the aryl in polystyrene. ${ }^{57}$ Samples nc-sPS-0.5, nc-sPS-2, nc-sPS-6 were exposed to the acetyl sulfate for 30 minutes, 2 hours, 6 hours, respectively. The length of the reaction time dictated the extent of the reaction and hence the ionic loading in the nc-sPS sample. Conversely, samples nc-P2VP-2, nc-P2VP-4, nc-P2VP-6 were exposed to methyl iodide 
(MeI) vapor for 2, 4, 24 hours to incorporate n-methylpyridinium iodide groups into the grafted P2VP polymer brushes. Illustrative representations of the nanoconfined polycation and polyanion brushes are given in Figure 1b. Figure 1c represents the chemical structures of the ncsPS and nc-mP2VP polymer electrolyte brushes. Figures S1a and S1b in the supporting information (SI) depict the chemical reaction for introduction of ionic groups to the tethered brushes. Figure S1c in the SI shows an illustration of the non-confined sPS and mP2VP polymer electrolyte brushes on the substrates. 
a.)

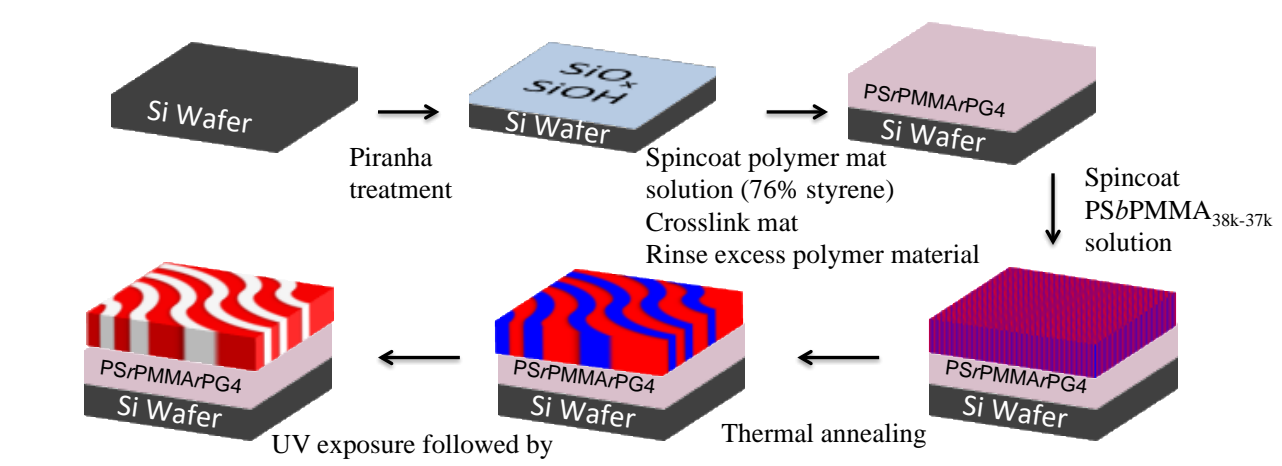

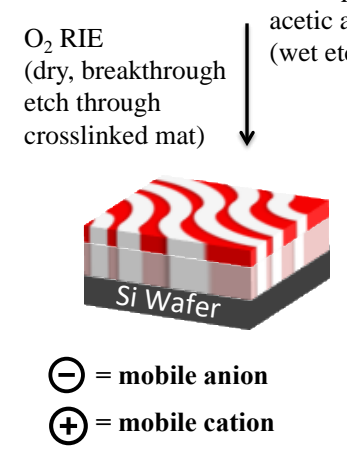
$\mathrm{O}_{2} \mathrm{RIE}$
(dry, breakthrough
etch through
crosslinked mat) $\downarrow$ (wet etch PMMA out) brush away acid rinse
tch PMMA out)

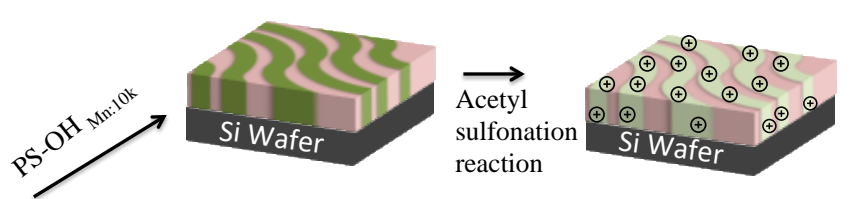

Backfill polymer Thermally graft Rinse excess brush

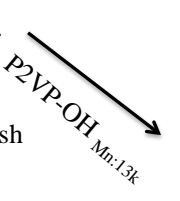

b.)

\section{Nano-confined polymer electrolyte brushes}
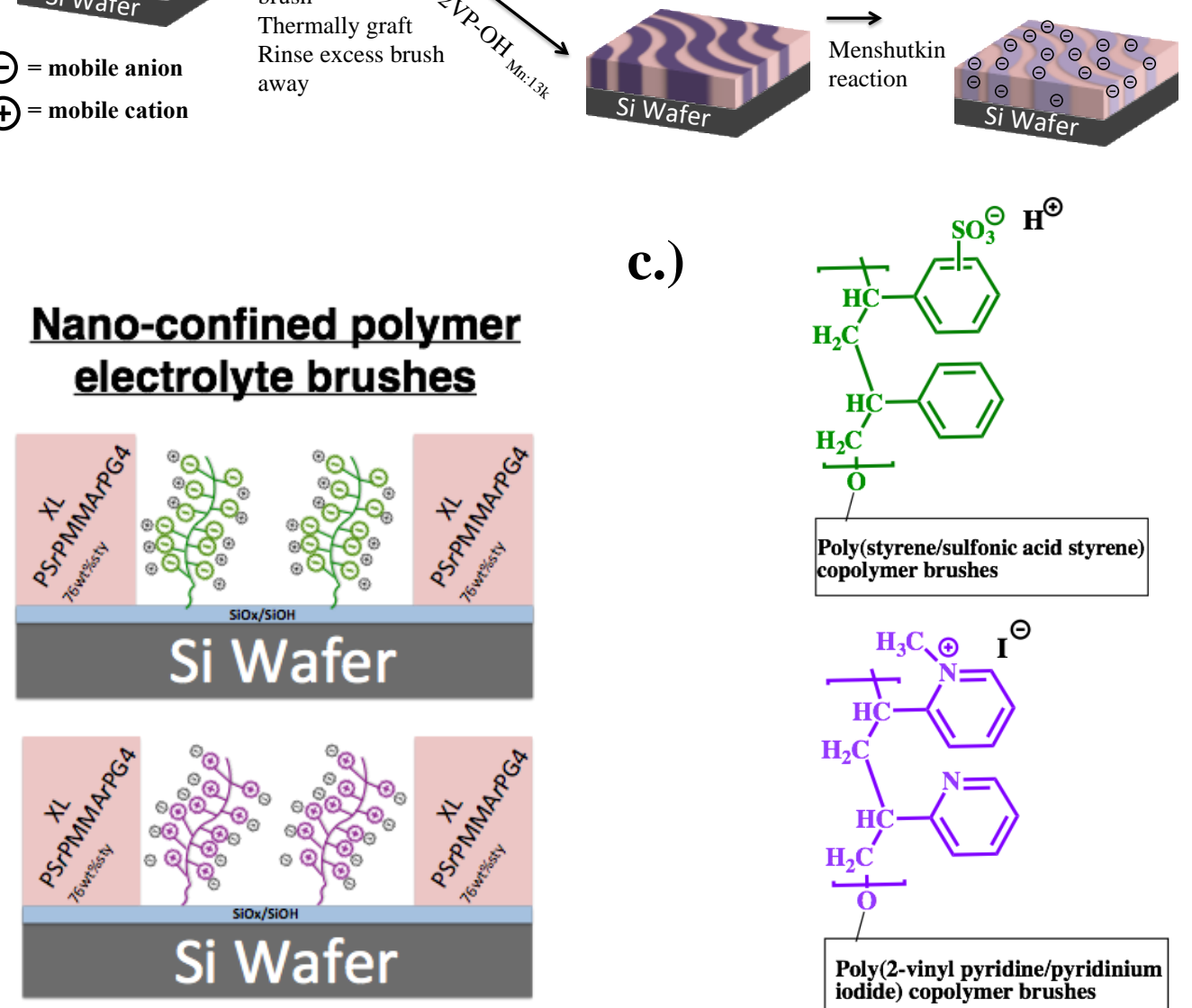
c.)

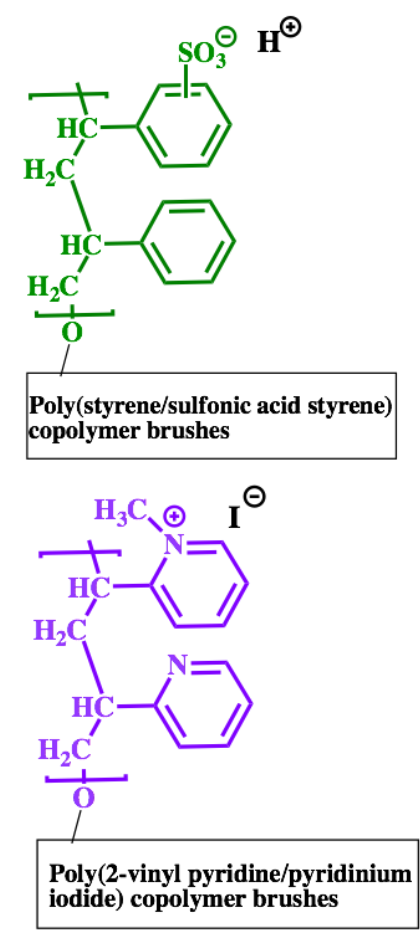

Figure 1. a.) Scheme to prepare nano-confined polycation and polyanion brushes from block copolymer templates; b.) Illustrations of nano-confined polycation and polyanion brushes; c.) Chemical structures of polycation and polyanion brushes. 
Figure 2a shows the SEM image of the self-assembled PS- $b$-PMMA $M n: 38 k-36.8 k$ fingerprint lamellae block copolymer. Both polymer blocks are oriented perpendicular to the substrate surface. The wet-etched and dry-etched PS- $b$-PMMA samples are shown in Figures $2 \mathrm{~b}$ and $2 \mathrm{c}$. The etching created nanosized gaps in the crosslinked mat. Figure $2 \mathrm{~d}$ is a SEM image of backfilled OH-PS brushes grafted in the gap regions and ionized with sulfonic acid moieties. Similarly, Figure 2e is a SEM image of backfilled OH-P2VP brushes grafted in the gap regions and ionized with n-methylpyridinium iodide moieties. The SEM images given in Figures $2 \mathrm{~d}$ and 2e correspond to nc-sPS-0.5 (exposed to acetyl sulfonate for $30 \mathrm{~min}$ ) and nc-mP2VP-24 (exposed to MeI vapor for 24 hours) polymer electrolyte brush samples, respectively. Zoomedout images of Figures 2a to 2e are provided in the SI as Figures S2 to S6. The zoomed-out images demonstrate the periodicity of the samples' structures over large areas. It is important to note that the processes of $\mathrm{i}$.) back filling the $\mathrm{OH}-\mathrm{P} 2 \mathrm{VP}$ and $\mathrm{OH}-\mathrm{PS}$ brushes in to the wet-etched and dry-etched block copolymer template and ii.) rinsing excess, unreacted $\mathrm{OH}-\mathrm{P} 2 \mathrm{VP}$ and $\mathrm{OH}-$ PS brushes caused full removal of the etched PS $b$ PMMA block copolymer because the original block copolymer film thickness with mat was $44 \mathrm{~nm}$, but the sample thickness values of nc-PS-0 and nc-P2VP-0 ranged from 14 to $18 \mathrm{~nm}$. Figures S7a and S7b shows that the height difference between the patterned crosslinked mat and the backfilled brushes (determined from AFM) in ncsPS-6 ranged from 0.5 to $5.5 \mathrm{~nm}$. The height different between the brush region and the crosslinked mat region was further evidence substantiating that the etched block copolymer template had been removed in the nano-confined samples.

The fingerprint lamellae images in Figures $2 \mathrm{~d}$ and $2 \mathrm{e}$ substantiate the pattern transfer of the self-assembled PS- $b$-PMMA block copolymer template into the underlying crosslinked mat leading to the creation of nano-confined, lamellae type structures of polyanion and polycation 
brushes. The images provided in Figure $2 \mathrm{~d}$ and $2 \mathrm{e}$ are representative for the nanoconfined polycation and polyanion brushes for all reaction times. However, the nc-sPS sample exposed to acetyl sulfonate for 6 hours (nc-sPS-6) slightly differed from the structure observed in Figure $2 \mathrm{~d}$ (see Figures S7a and S7b in the SI for SEM and AFM images). Swollen block regions in Figures S7a and S7b appeared in the fingerprint lamellae suggesting sulfonation of the crosslinkable mat material. At long reaction times (e.g, 6 hours), the acetyl sulfonate reagent is able to penetrate the crosslinked domain and sulfonate the styrene groups. For shorter reaction times, the acetyl sulfonate reacts first with the non-crosslinked backfilled polystyrene brushes because of the lower mass transfer resistance when compared to the crosslinked region.

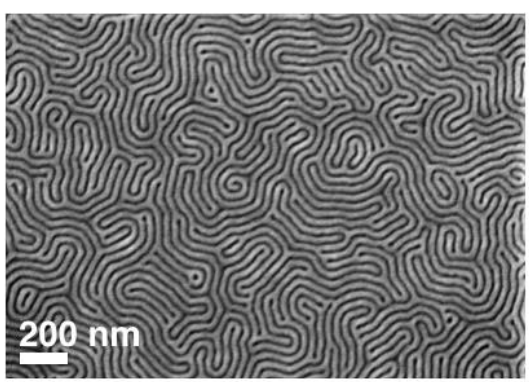

a.) Pristine self-assembled PSbPMMA

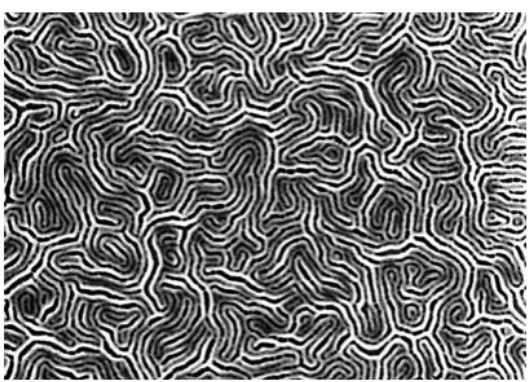

b.) Wet etched PMMA out of BCP template

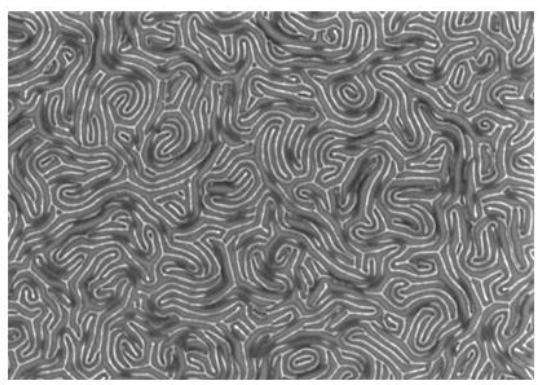

c.) Dry, breakthrough etch of crosslinked mat

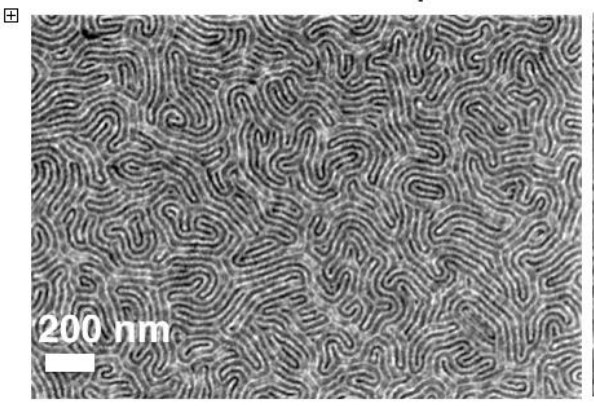

d.) Backfilled OH-PS brush, thermally grafted, and sulfonated (30 $\mathrm{min})$

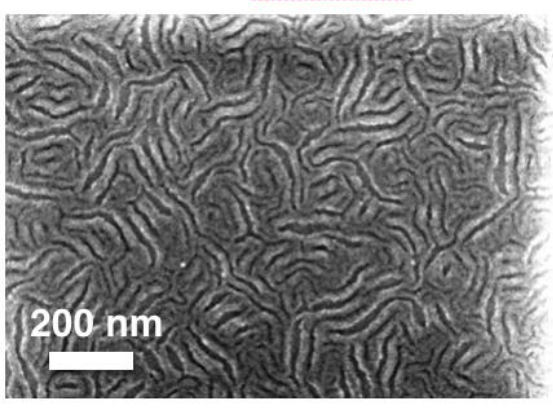

e.) Backfilled OH-P2VP brush, thermally grafted, and methylated

Figure 2. SEM images of a.) Self-assembled, lamellae forming PS- $b$-PMMA $\mathrm{A}_{M n: 38 k-36.8 k} \mathrm{BCP}, \mathrm{b}$.) Wet-etched PMMA domains from the PS- $b$-PMMA ${ }_{M n: 38 k-36.8 k}$ BCP template, c.) Oxygen reactive ion etching of the non-preferential, crosslinked mat underneath the BCP and trim etch of the PS domains, d.) Backfilled and thermally grafted OH-PS brushes into the patterned areas followed by acetyl sulfonation of the PS brushes for 30 minutes (sample nc-sPS-0.5), e.) Backfilled and thermally grafted OH-P2VP brushes into the patterned areas followed by methylation of the P2VP brushes for 24 hours (sample nc-mP2VP-24). 
Figures $3 \mathrm{a}$ and $3 \mathrm{~b}$ provide the FTIR spectra of nc-sPS and nc-mP2VP polymer electrolyte brushes before and after addition of ionic groups. The vibrational stretching mode associated with $\mathrm{S}-\mathrm{O}$ in $-\mathrm{SO}_{3}{ }^{-}$was observed in the nc-sPS samples at $1040 \mathrm{~cm}^{-1}$, while the phenyl- $\mathrm{SO}_{3}{ }^{-}$ stretching mode was observed at $1130 \mathrm{~cm}^{-1}$. The broad stretching peak observed from 1180 to $1300 \mathrm{~cm}^{-1}$ corresponded to asymmetric stretching of S-O in $-\mathrm{SO}_{3}{ }^{-}$(see Figure 3a). ${ }^{57,58}$ These signals demonstrated greater intensity with increasing exposure time to acetyl sulfonate. The two small peaks at $1490 \mathrm{~cm}^{-1}$ and $1450 \mathrm{~cm}^{-1}$ in nc-PS, nc-sPS-0.5, and nc-sPS-2 correspond to the carbon-carbon stretching in the aromatic ring of PS. ${ }^{59}$ These two peaks become a large and broad peak from $1500 \mathrm{~cm}^{-1}$ to $1450 \mathrm{~cm}^{-1}$ in nc-sPS- 6 because of the distortion of the carbon-carbon stretching due to the nearby sulfonate moiety in the aromatic ring. $\mathrm{C}-\mathrm{N}^{+}$stretching was observed at $1620 \mathrm{~cm}^{-1}$ for nc-P2VP samples exposed to MeI vapor substantiating the conversion of the pyridine groups to n-methyl pyridinium iodide - anion charge carriers. ${ }^{15}$ The signal at $1350 \mathrm{~cm}^{-1}$ in the nc-P2VP and nc-mP2VP samples was attributed to the carbon-carbon stretching in the pyridine ring ${ }^{60}$ Hi-res XPS spectra for sulfur $2 \mathrm{p}$ nc-PS/nc-sPS-6 and for nitrogen $1 \mathrm{~s}$ ncP2VP/nc-mP2VP-24 are given in Figures S8a and S8b in the SI. Figure S8a shows a sulfur 2p signal at $169 \mathrm{eV}$ that is absent in the nc-PS sample not exposed to acetyl sulfonate. Similarly, Figure S8b displays a new nitrogen 1s signal at $404 \mathrm{eV}$ that corresponds to the quaternarized nitrogen in nc-mP2VP-24.

In addition to spectroscopic evidence, water contact angle measurements of the different nano-confined polymer and polymer electrolyte brushes supported the introduction of ionic moieties. The introduction of hydrophilic ionic moieties into the nc-PS and nc-P2VP brushes demonstrated a reduction in the brushes' water contact angle. The reduction in water contact angle was proportional to the exposure time with the reagents that introduced ionic groups (see 
Figures $3 \mathrm{c}$ and $3 \mathrm{~d}$ for water contact angle values of the nanconfined samples and Figures S9a and S9b in the SI for water contact angle values of brush samples that completely cover the substrate).

FTIR spectra, XPS spectra, and water contact angle confirmed the presence of ionic groups within the nanoconfined polymer brushes. Tethered ionic moieties along the polymer backbone are hydroscopic rendering a lower interfacial energy difference between the condensed water droplet and the grafted brush with larger ionic loadings.

It is important to discuss the water contact angle value of nc-sPS exposed to acetyl sulfonate for 6 hours (i.e., nc-sPS-6) in Figure 3c. This sample almost displayed complete water spreading. The water contact angle value of nc-sPS-6 in Figure 3c was similar to the values of sPS brushes and mP2VP brushes (polymer electrolyte brushes homogenously distributed over the surface - see Figure S9 in the SI). Because nc-sPS-6 displayed water contact angle values that are similar to sPS samples exposed to acetyl sulfonate for 6 hours (sPS-6), it was inferred that the crosslinked, patterned domains also underwent sulfonation because these domains contained styrene repeat units. Sulfonation of the crosslinked mat material, in addition to the PS brush, fostered complete water spreading. To support the assertion that sulfonation of styrene in the crosslinked mat material occurred but at a slower rate, it is important to mention that nc-sPS0.5 and nc-sPS-2 did not display the same drop in water contact angle from its non-ionized variant as the sPS-0.5 and sPS-2 did from its non-ionized variant. The water contact angle data, in conjunction with the swollen crosslinked mat domains of the nc-sPS-6 observed via SEM and AFM images (see Figures S7a and S7b in the SI), support that the crosslinked mat material was subject to sulfonation; however, at a slower rate when compared to the PS homopolymer brush 
because the nanopatterned mat contained crosslinked domains that had a greater barrier to the mass transfer of acetyl sulfonate.

a.)

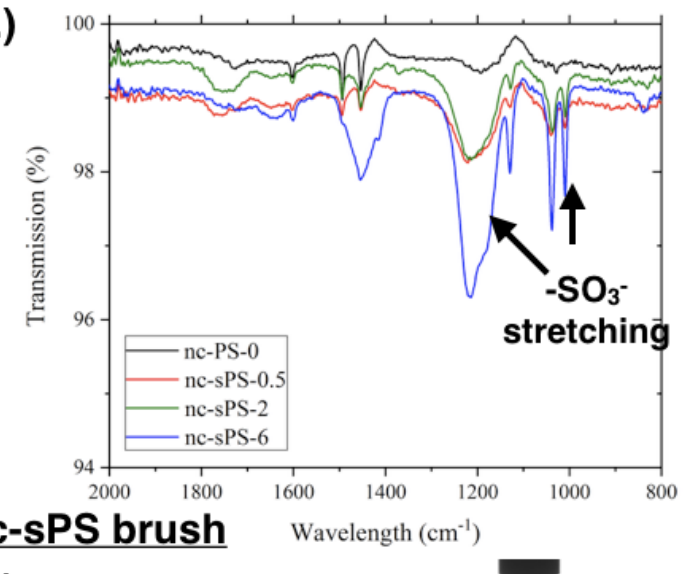

c.)
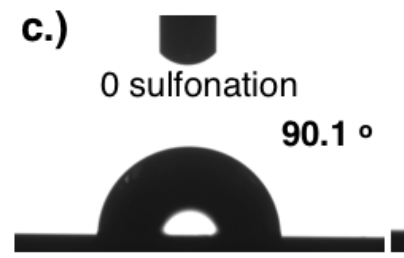

nc-P2VP brush

d.)

$0 \mathrm{hr}$ methylation

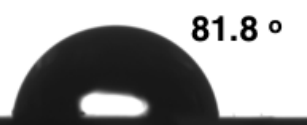

$72.3^{\circ}$
$2 \mathrm{hr}$ methylation

$76.2 \circ$ b.)

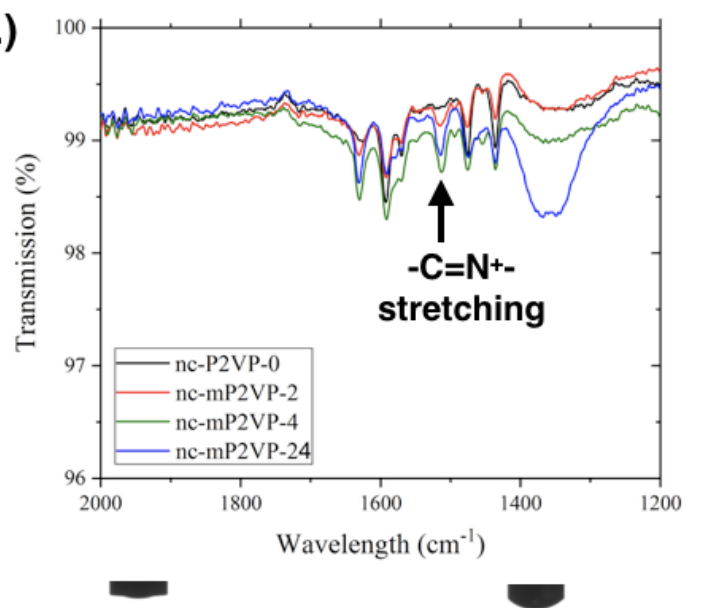

2hr sulfonation

70.1 。

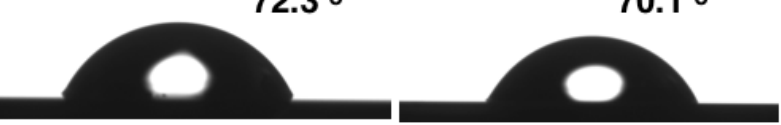

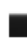

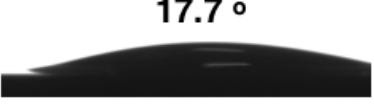

6hr sulfonation

17.7 。

$24 \mathrm{hr}$ methylation

$4 \mathrm{hr}$ methylation

\begin{abstract}
Figure 3. FTIR spectra of a.) nc-PS and nc-sPS samples with different acetyl sulfonation times and b.) nc-P2VP and nc-mP2VP samples with different methylation times; Water contact angle values of c.) nc-PS and nc-sPS samples with different acetyl sulfonation times and d.) nc-P2VP and nc-mP2VP samples with different methylation times.
\end{abstract}

Figures $4 \mathrm{a}$ and $4 \mathrm{~b}$ are the Nyquist plots from EIS experiments to determine the in-plane resistance values of the nano-confined samples. These resistance values were used to calculate the film ionic conductivity values reported in Figures $4 \mathrm{c}$ and $4 \mathrm{~d}$. The in-plane resistance values were determined by formulating an electric circuit equivalent model that fitted the data in the Nyquist plot (same model reported in our previous work ${ }^{15}$ - which is also provided in Figure S10 in the SI section). The ionic conductivity of the nc-sPS and nc-mP2VP samples reached nearly 
$160 \mathrm{mS} \mathrm{cm}^{-1}$ and $25 \mathrm{mS} \mathrm{cm}^{-1}$ - both high values making them excellent candidates for emerging electrochemical devices featuring thin film polymer electrolytes (e.g., thermal galvanic and nonvolatile memory devices) ${ }^{7-10}$. It should be noted that the significantly lower ionic conductivity of the $\mathrm{mP} 2 \mathrm{VP} / \mathrm{nc}-\mathrm{mP} 2 \mathrm{VP}$ samples compared to sPS/nc-sPS samples is attributed to the disparate intrinsic mobility values of the counterions. The iodide counterion has one fifth the ionic mobility of hydronium in infinitely diluted water at $25{ }^{\circ} \mathrm{C} .{ }^{1}$ The large ionic mobility difference is mainly due to hydronium breaking and forming bonds with water and migrating via the Grotthuss shuttling mechanism, where the iodide counterion can only migrate via the vehicular mechanism.
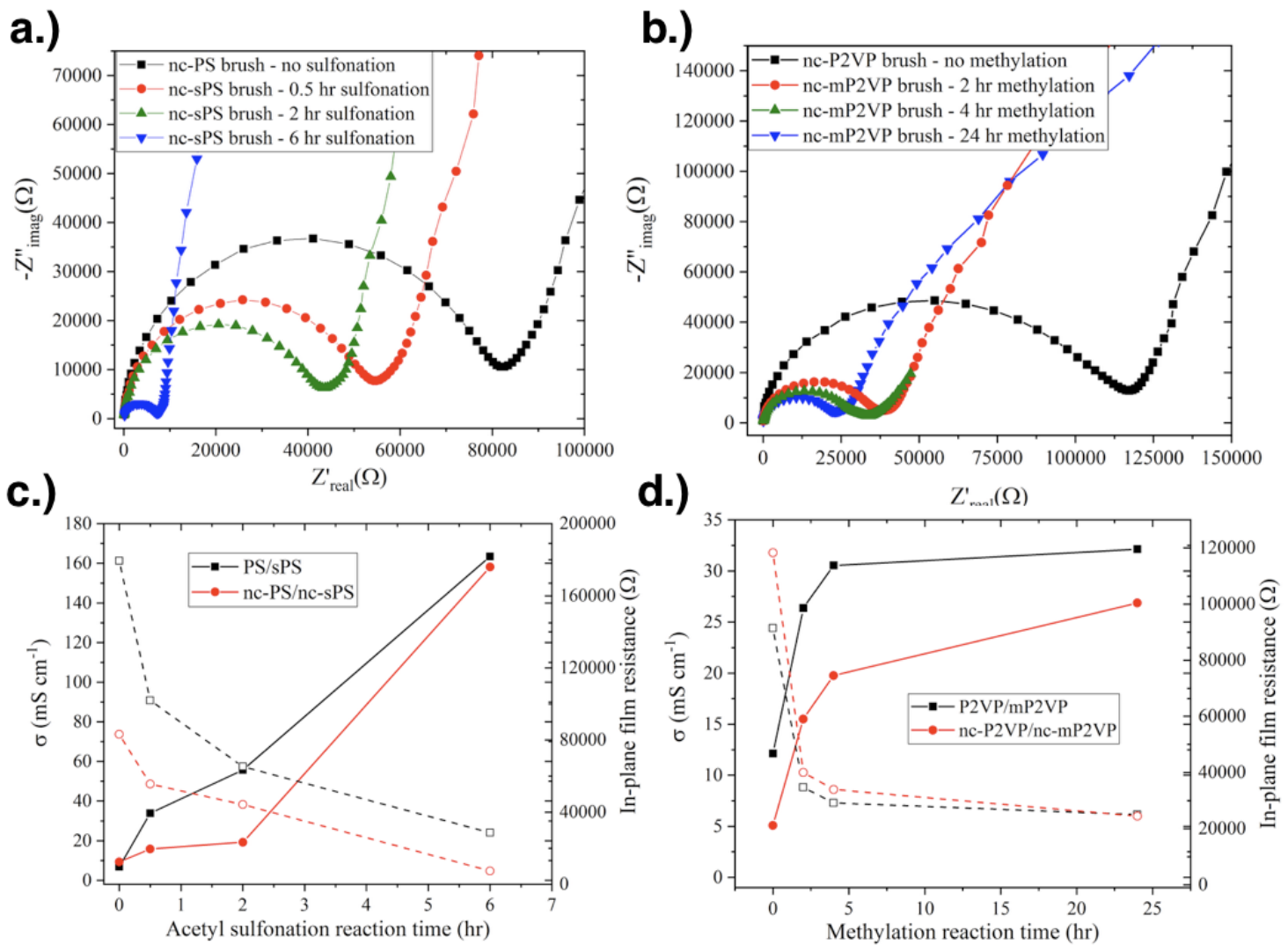

Figure 4. Nyquist plots of a.) nc-PS and nc-sPS samples with different reactions times with acetyl sulfonate and b.) nc-P2VP and nc-mP2VP films with different exposure times to MeI. The determined in-plane resistance (dashed lines) and ionic conductivity (solid lines) for c.) nc-PS, nc-sPS, PS, and sPS films for different reactions times with acetyl sulfonate and d.) nc-P2VP, nc-mP2VP, P2VP, and mP2VP films with different exposure times to MeI. 
Table 1 reports the samples' thickness values used to calculate the in-plane resistance. For the PS and nc-PS samples, the film thickness became smaller when introducing the sulfonic acid groups. The film thickness continued to decrease with larger ionic loadings for the sPS and nc-sPS samples. The extent of reduction was more significant for the sPS samples. The reduction in sample thickness upon introduction of sulfonate groups indicated the possibility of counterion condensation within these samples, because brush shrinkage/collapse has been well documented for polymer electrolyte brushes experiencing counterion condensation ${ }^{61-66}$. Figure 5 a depicts the reduction in polymer brush height upon introduction of ionic charges for nano-confined and nonconfined samples. Similar to sPS and nc-sPS samples, the nc-mP2VP brushes also displayed a small drop in thickness with introduction of ionic charges. It should be noted that a conflicting trend was observed for the $\mathrm{P} 2 \mathrm{VP} / \mathrm{mP} 2 \mathrm{VP}$ samples. The film thickness values for the nonconfined P2VP slightly increased upon methylation because mass was being added to this film. However, a $40 \%$ conversion of the pyridine groups to n-methylpyridinium iodide, which was observed in our previous study ${ }^{11}$ for MeI exposure for 24 hours, would result in a mass increase of $54 \%$. Assuming that the free volume in the polymer is small and the mass gain would be commensurate with film thickness increase, the anticipated film thickness for mP2VP-24 should be $14.8 \mathrm{~nm}$ (based on P2VP-0 sample thickness being $9.6 \mathrm{~nm}$ ). However, the film thickness value of mP2VP-24 is $13.2 \mathrm{~nm}$. The smaller gain in thickness value of mP2VP-24 despite the large mass uptake is attributed to a partial brush collapse by the introduction of ionic moieties into the polymer brushes. In a subsequent section, the atomistic molecular dynamics (MD) simulations will demonstrate that mP2VP samples display less counterion condensation versus sPS samples. As a result, it was not expected that the mP2VP samples would shrink as much as the sPS samples. The difference in the extent of counterion condensation for sPS/nc-sPS versus 
$\mathrm{mP} 2 \mathrm{VP} / \mathrm{nc}-\mathrm{mP} 2 \mathrm{VP}$, informed from the simulations, is ascribed to the type of counterion and its level of hydration. To recap this section, the reduction of thickness values for polymer brushes post-introduction of ionic moieties is consistent with previous results of polymer electrolyte brushes and counterion condensation. ${ }^{61-66}$

Table 1. Sample thickness values determined by ellipsometry

\begin{tabular}{|ccccc|}
\hline Sample & nc-PS-0 & nc-sPS-0.5 & nc-sPS-2 & nc-sPS-6 \\
Thickness $(\mathrm{nm})$ & 13.7 & 12.0 & 12.5 & 9.0 \\
Sample & PS-0 & sPS-0.5 & sPS-2 & sPS-6 \\
Thickness $(\mathrm{nm})$ & 8.6 & 3.1 & 2.9 & 2.3 \\
& & & & \\
Sample & nc-P2VP-0 & nc-mP2VP-2 & nc-mP2VP-4 & nc-mP2VP-24 \\
Thickness (nm) & 17.6 & 17.1 & 15.8 & 16.1 \\
Sample & P2VP-0 & mP2VP-2 & mP2VP-4 & mP2VP-24 \\
Thickness (nm) & 9.6 & 11.6 & 11.9 & 13.2 \\
\hline
\end{tabular}

*Note: Original thickness of the PS $b$ PMMA block copolymer film was $44 \mathrm{~nm}$.

The in-plane electrical resistance values offer further evidence to the support the premise of counterion condensation occurring within these samples. The nano-confined polymer electrolyte brushes yielded about the same, or lower, in-plane resistance values when compared to the non-confined samples. Because the nc-sPS samples did not display the same reduction in film thickness as the non-confined samples, it was hypothesized that less counterions were condensed and the greater dissociation of the counterions promoted diffusion subsequently resulting in lower in-plane resistance values (see Figure S11 in the SI section for a depiction on condensed counterions and ionic transport). From our experiments, the nc-sPS samples were always at least $20 \mathrm{k} \Omega$ lower than the sPS samples. The in-plane resistance values of the ncmP2VP samples were about the same for all mP2VP samples despite it having only half of its volume fraction available to transport ions. It is important to consider that the nano-confined samples contain disconnected ionic domains in their lamellae structure that are known to compromise ionic conductivity. ${ }^{15}$ The ionic conductivity values of the non-confined samples are 
inflated in Figures $4 \mathrm{c}$ and $4 \mathrm{~d}$ because of the samples' smaller thickness values - which occurred upon the introduction of the ionic groups. In other words, relying on the ionic conductivity data for this analysis is misleading. The ionic conductivity values are provided for comparing with other results in the literature. The lower/equivalent in-plane electrical resistance values of the nano-confined samples indicated that the nano-confined brushes were facilitating greater rates of counterion transport because they were less susceptible to counterion condensation.

To further substantiate that counterion condensation was at play in these systems, 2D AFM force mapping and atomistic MD simulations were employed. Figure 5a illustrates a nanoconfined and non-confined polyanion brushes before and after introduction of charges. The nano-confined brushes are depicted to show less shrinkage upon introduction of charge because they are less susceptible to counterion condensation (note: it is expected that a polycation brush would display the same behavior). Manning's Theory of Counterion Condensation ${ }^{67}$ (see Equation 1) states that polymer electrolytes will continue to condense their counterions to reduce their overall charge density and to a critical value (which is 1 for the Manning parameter, $\xi$, if a monovalent salt and tethered ionic group is used). It is believed that the nano-confined system consisting of periodic structures of charged and non-charged domains reduces the overall charge density mitigating the condensation effect. But, it will also be shown later on that water solvation and selection of counterion type can also influence the extent of counterion condensation. In the subsequent paragraphs below, the 2D AFM force mapping results supported that counterion condensation was occurring within these materials and that it manifests at a greater degree in the non-confined polymer electrolyte brushes versus the nano-confined polymer electrolytes. Atomistic MD simulations also supported the differences in counterion condensation for nanoconfined and non-confined samples. These simulations quantified the amount of counterions 
condensed and the vehicular diffusion coefficients for ion transport for a simulation box that consisted of idealized polymer brushes flooded with water (note: the simulations did not account for other modes of ionic transport, such as Grotthuss shuttling mechanism, which is a priority in our future work). The 2D AFM force mapping results will be discussed first.

Manning's Theory of Counterion Condensation:

$$
\xi=\frac{e^{2}}{4 \pi \varepsilon_{0} \varepsilon k T b}=\frac{\lambda_{b}}{b}
$$

$\xi$ : Manning parameter

$e$ : protonic charge

$\varepsilon$ : solvent dielectric constant

$\varepsilon_{0}$ : vacuum permittivity

$k$ : Boltzmann's constant

$T$ : temperature

$b$ : average distance between charges on the polymer electrolyte chain

$\lambda_{\mathrm{b}}$ : Bjerrum length 
a.)

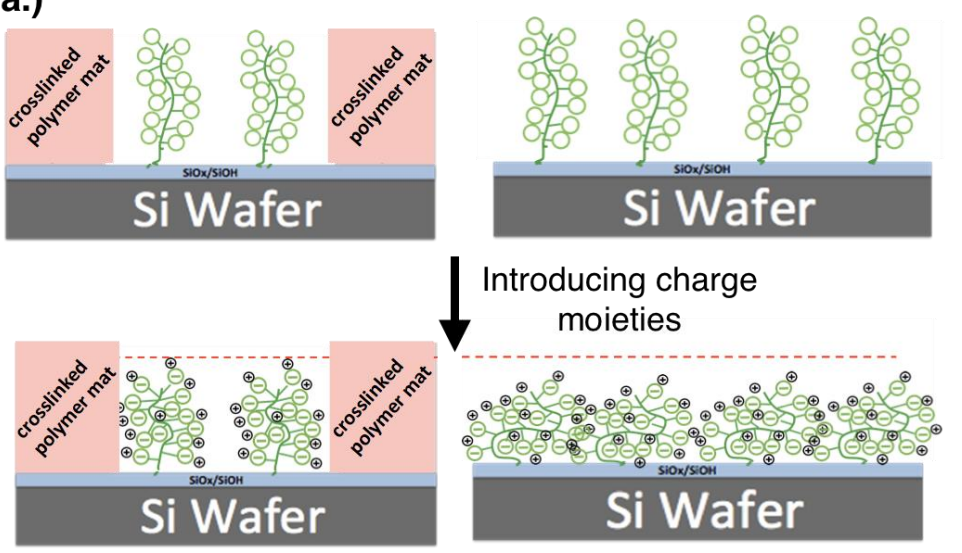

b.)

c.)

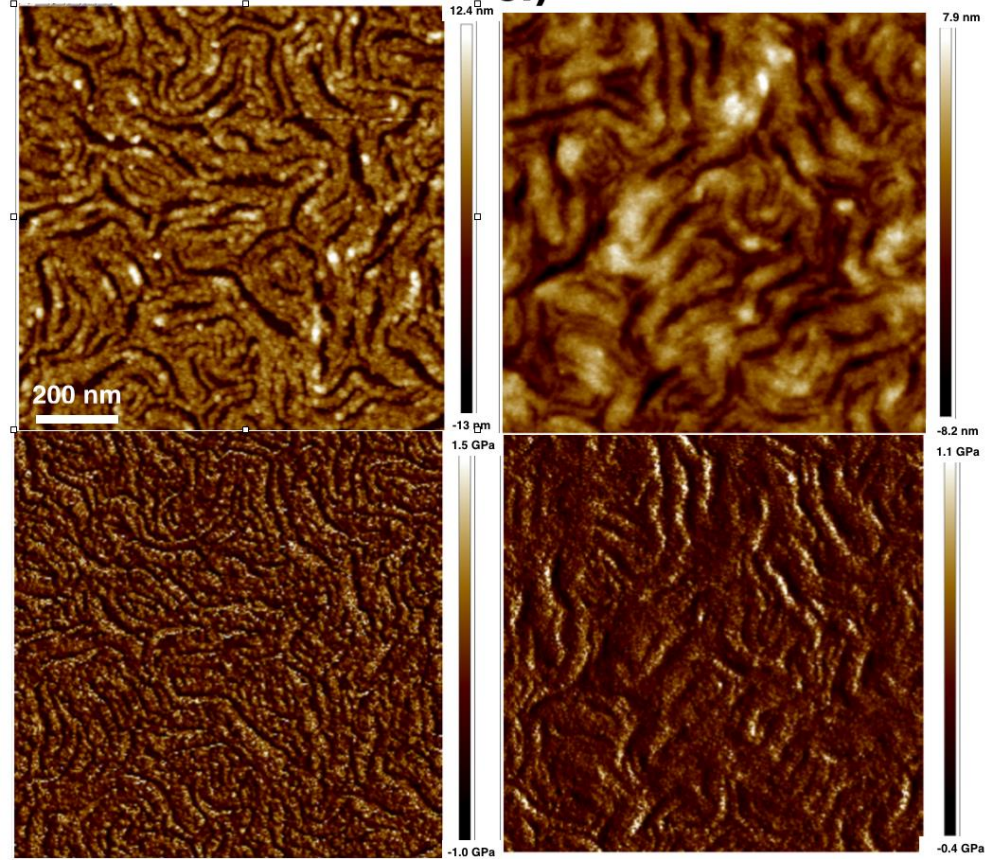

d.)

e.)
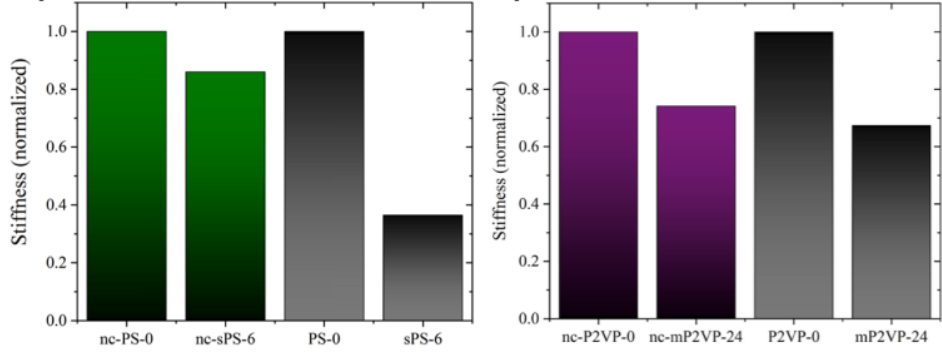

Figure 5. a.) Cartoon representation of the polymer brush thickness in a nano-confined and nonconfined regions before and after introduction of tethered anionic moieties; 2D AFM force mapping images of b.) nc-PS and c.) nc-sPS-6. Top images correspond to height and bottom images corresponds to modulus; the relative stiffness values of d.) nc-sPS-6 and sPS-6 and e.) nc-mP2VP-24 and mP2VP-24 normalized against their non-ionic variants. 
Balastre et al. ${ }^{50}$ used a surface force apparatus to quantify counterion condensation in grafted polycation and polyanion brushes interfaced with different liquid salt solution concentrations. It has been shown by Tirrell and co-workers ${ }^{49}$ that surface force measurements by an AFM can also provide similar type of information when compared to a surface force apparatus instrument. Figures $5 \mathrm{~b}$ to $5 \mathrm{c}$ provide representative height and modulus (force map) AFM images for nc-PS 0 (Figure 5b) and nc-sPS-6 (Figure 5c). The top panel in Figures 5b and $5 \mathrm{c}$ are the height images and the bottom panel corresponds to the force map images (the output is the modulus of the sample that is computed later on to stiffness values as described in the SI). Figure $5 \mathrm{~d}$ and $5 \mathrm{e}$ compare the stiffness value (normalized to the non-ionic variant of the sample) for the nano-confined and non-confined polymer brushes after introduction of ionic groups. The stiffness values have the units of force per length and are calculated based on the approach and retraction of the AFM tip during force mapping measurements. Because the $2 \mathrm{D}$ force maps take a long time to acquire, the samples with no ionic groups and the largest concentration of ionic groups were prioritized. Figures $5 \mathrm{~d}$ and $5 \mathrm{e}$ demonstrate that the sample stiffness is reduced upon introduction of ionic groups into the polymer brushes. The nano-confined samples yielded a smaller drop in relative stiffness when compared to the non-confined samples: 14 to $25 \%$ for the nano-confined samples and 33 to $65 \%$ for the non-confined samples. These results indicated that the nano-confined samples were better at retaining their mechanical integrity upon introduction of ionic moieties. Balastre et al..$^{50}$ adopted mean-field scaling theories to examine polymer electrolyte brush forces and stretching based on a balance of elastic and osmotic forces. Two regimes were used to describe force versus distance curves for approach and retraction with a surface force apparatus. Because our experiments were not carried out in liquid solutions with dissolved salt, the osmotic regime force only applies. It has been shown that the force-distance 
curves $\left(\mu \mathrm{N} \mathrm{m}^{-1}\right)$ for the osmotic regime are directly proportional to alpha, which is the fraction of non-condensed counterions per monomer unit, for the mechanical equation of state. Hence, the force exerted by the brush is compromised to a greater extent for a brush containing more condensed counterions - which is what is observed in our data set when comparing the nonconfined samples versus the nano-confined samples. The 2D AFM results support the premise that the nano-confined polymer electrolyte brushes are less susceptible to counterion condensation.

To further corroborate if nano-confined samples displayed different extents of counterion condensation versus non-confined samples, atomistic MD simulations were performed for idealized systems. Four atomistic molecular simulations were carried out using a conventional non-reactive force field using OPLSAA ${ }^{68}$ (the SI section provides greater detail about the simulation methodology). This force field does not consider the Grotthuss shuttling mechanism or proton transfer events. The Grotthuss shuttling mode of transport will be examined for condensed and non-condensed ions in future work. One set of simulations consisted of four charged polymer electrolyte brushes aligned along one axis with a periodic spacing of $17 \AA$. For the nano-confined samples, these brush polymer electrolyte brushes were separated by $140 \AA$ from four non-charged polystyrene brushes. The $140 \AA$ Aistance represents the half-period of PS-

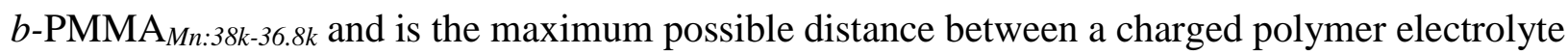
and a non-charged polymer domain in the experimental system. The rationale for the large separation of polymer brushes was based upon the desire to clearly observe and quantify the water distribution at the end of the molecular dynamics simulation. The distribution of water in the system was shown to have an impact on the distribution of condensed and non-condensed counterions. Figure 6a, which is for nc-sPS, provides a representative configuration of the 
idealized polymer brushes simulated. For the non-confined samples, the simulation setup replaced the non-charged polymer brushes with identical polymer electrolyte brushes used in the nano-confined simulations (i.e., the non-confined simulation consists only of charged polymer electrolyte brushes). The polymer brushes for all simulations consisted of ten monomeric repeat units with the brushes attached to a SiOx substrate. If the polymer brush contained charge, all of the monomeric units were ionized. The initial simulation box had a length of $45 \AA$ in the zdirection (perpendicular to the substrate) and was solvated with water uniformly before the start of the simulation after which the boxlength was extended to $100 \AA$ in the z-direction, resulting in two interfaces namely substrate-polymer brushes/water and polymer brushes/water-air. The molecular dynamics simulations were equilibrated for 5 nanoseconds followed by a 20 nanoseconds production run with 1 femtosecond time steps. Further details are provided in the supporting information in addition to a movie showing the simulation for the idealized nc-sPS. The simulation outputs were the fraction of condensed and non-condensed counterions, the average diffusion coefficients of all counterions, and the diffusion coefficient of the noncondensed countersions (see Table 2). Figures $6 \mathrm{~b}$ to $6 \mathrm{e}$ shows the final distribution of water at the end of the molecular dynamics in the very idealized nano-confined and non-confined polymer electrolyte brushes.

Table 2 highlights that the nano-confined polymer electrolyte brushes were less prone to counterion condensation. It also shows that the nano-confined polymer electrolyte samples have higher average vehicular diffusion coefficient of the counterions over non-confined samples. Hence, reduced counterion condensation increases the average vehicular diffusion coefficient for the counterions and explains why smaller (or relatively equivalent) bulk electrical resistance values were obtained for nano-confined samples versus non-confined samples from experiments 
using IDE setups. Table 2 also shows that the free counterions had on average the same diffusion coefficient values for vehicular transport. In reality, the ionic mobility for hydronium counterions is about $5 \mathrm{x}$ larger than iodide counterions, but these simulations did not take into account the Grotthuss shuttling mechanism. This shuttling mechanism enhances hydronium ionic mobility by a factor of $4 .{ }^{69}$ It is interesting to note that the non-condensed counterion diffusion coefficient values are the same for all systems simulated, but the average diffusion coefficient values for the nano-confined samples were higher than the non-confined samples. This result is directly attributed to the different fraction of condensed counterions within the idealized system and that the condensed counterions have substantially smaller diffusion coefficients. Interestingly, the ncsPS and sPS samples from the simulations had $77 \%$ and $84 \%$ of their hydronium counterions condensed, respectively. These results align with the works by Beers and Balsara, which showed $78 \%$ to $92 \%$ of hydronium in Nafion ${ }^{\circledR}$ (a perfluorosulfonic acid membrane) were condensed ${ }^{41}$, and Balastre et al. ${ }^{50}$ that showed that sulfonated polystyrene brushes with sodium counterions were 80 to $85 \%$ condensed.

It is important to highlight that the simulations revealed that water solvation and counterion type had an impact on the extent of counterion condensation. The fraction of counterions condensed in the polycation system (nc-mP2 VP and mP2VP) was smaller than the polyanion system (nc-sPS and sPS). However, less counterion condensation occurred in nc$\mathrm{mP} 2 \mathrm{VP}$ versus mP2VP. Figures $6 \mathrm{~b}-\mathrm{e}$ depict the distribution of water in the nano-confined and non-confined polymer electrolyte brushes at the end of the molecular dynamics simulations for the idealized systems. The nano-confined samples show a larger concentration of water in the charged domains than the non-confined samples as the non-charged domains are hydrophobic and guide water to the charged domains. This was more pronounced for the nc-sPS system 
versus the nc-mP2VP. The iodide counterion in nc-mP2VP is less hydrophilic when compared to the hydronium counterion. Thus, the water uptake was not heavily favored to the poly(nmethylpyridinium iodide) brushes versus non-charged brushes.

As previously mentioned and informed from Manning's Theory of Counterion Condensation (see Equation 1) 43, $^{47}$, it was hypothesized that the nano-confined domains would yield less counterion condensation because the nano-confined samples reduce the charge density of polymer electrolyte chains in the local vicinity. However, the simulation results shown in Figures $6 \mathrm{~b}-\mathrm{e}$ emphasize the importance of water content and solvation on counterion condensation. Manning's equation does not directly capture water content, but it could be accounted for in the dielectric constant that is used to compute the Bjerrum length in Equation 1. More water in the charged domain reduces the Bjerrum length lowering the Manning parameter that normalizes the Bjerrum length over the average distance between fixed charges on the polymer chain. In other words, fewer counterions need to condense to satisfy the critical value constraint.

Table 2. Atomistic molecular dynamics simulation results

\begin{tabular}{|cccc|}
\hline $\begin{array}{c}\text { Idealized samples } \\
\text { used in the } \\
\text { simulation }\end{array}$ & $\begin{array}{c}\text { Fraction of } \\
\text { counterions } \\
\text { condensed }\end{array}$ & $\begin{array}{c}\text { aAverage } \\
\text { counterion } \\
\text { diffusion coefficient } \\
\left(\mathbf{c m}^{2} / \mathbf{s}\right)\end{array}$ & $\begin{array}{c}\text { Non-condensed } \\
\text { counterion diffusion } \\
\text { coefficient }\left(\mathbf{c m}^{2} / \mathbf{s}\right)\end{array}$ \\
sPS & 0.84 & $0.73 \times 10^{-5}$ & $5.2 \times 10^{-5}$ \\
nc-SPS & 0.77 & $1.09 \times 10^{-5}$ & $5.2 \times 10^{-5}$ \\
mP2VP & 0.75 & $1.82 \times 10^{-5}$ & $5.3 \times 10^{-5}$ \\
nc-P2VP & 0.72 & $1.90 \times 10^{-5}$ & $5.1 \times 10^{-5}$ \\
\hline
\end{tabular}

${ }^{a}$ This diffusion coefficient is the average for both condensed and non-condensed counterions (weighted by the fraction of condensed and non-condensed counterions in the system) 

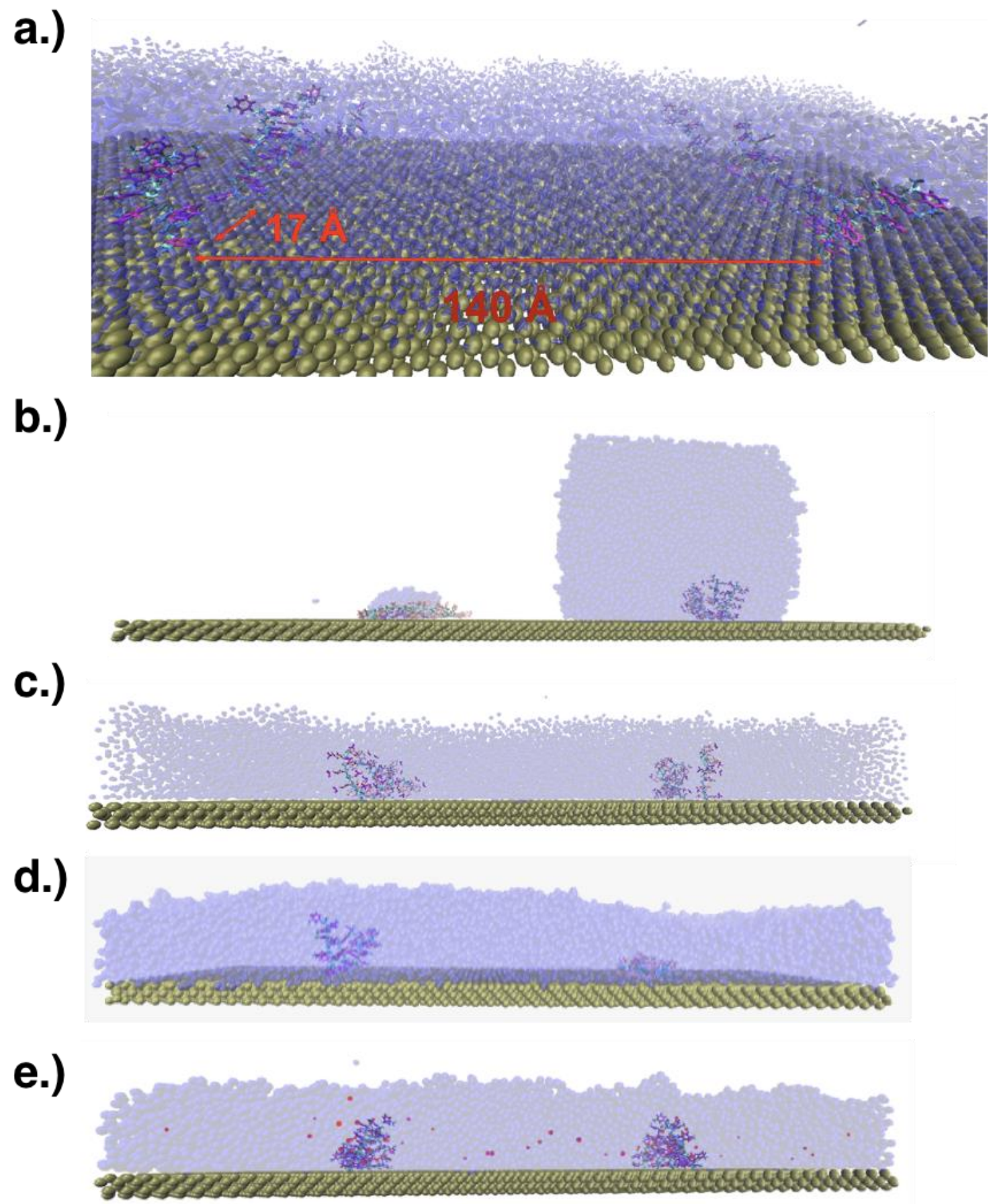

Figure 6. Representative snapshots from atomistic molecular dynamics simulations of nonconfined and nano-confined polymer electrolyte brushes on silicon substrate (olive green); a.) Snapshot of the system at the start of the atomistic molecular dynamics simulation; The distribution of water at the end of the molecular dynamics simulation in the idealized model systems b.) nc-sPS; c.) sPS; d.) nc-mP2VP; and e.) mP2VP. *Note: The polymer chain configurations shown here are idealized and a result of their interaction with water. The polystyrene chains for nano-confined samples repel water and thus collapse. Because these chains do not feature ionic groups they cannot condense ions and their depicted collapse is not due to counterion condensation. 


\section{CONCLUSIONS}

Block copolymer lithography was leveraged to prepare nano-confined polycation and polyanion brushes. FTIR, XPS, and water contact angle measurements substantiated the presence of ionic moieties in nano-confined and non-confined polymer electrolyte brushes. The samples displayed high ionic conductivity - in some cases well over $100 \mathrm{mS} \mathrm{cm}^{-1}$ for the hydronium conductors and over $20 \mathrm{mS} \mathrm{cm}^{-1}$ for the iodide anion conductors. The unexpected brush thickness and electrical resistance results hinted that counterion condensation was occurring in the nano-confined and non-confined polymer electrolyte brushes. 2D AFM force mapping, combined with atomistic MD simulations, substantiated the counterion condensation effect in the nano-confined and non-confined polymer electrolyte brushes. These techniques revealed that less counterion condensation occurred in the nano-confined polymer electrolyte brushes and as a result promoted greater counterion diffusion on average. The results motivate future studies to investigate counterion condensation on ionic transport in nano-confined media using a broad range of advanced metrology tools combined with molecular simulations.

\section{ACKNOWLEDGEMENTS}

This work was supported by the U.S. Department of Energy, Office of Science, Basic Energy Sciences Award \#DE-SC0018989 (PIs Arges \& Kumar). Y. K. was supported by the National Science Foundation Graduate Research Fellowship under Grant \#DGE-1144082. Finally, we acknowledge the MRSEC Shared User Facilities at the University of Chicago (NSF DMR-1420709) for use of the Asylum MFP3D AFM and Carl Zeiss Merlin SEM. RK and KL are grateful to LSU-HPC and Louisiana Optical Network Initiative for computer time. 


\section{SUPPORTING INFORMATION}

The supporting information provides the procedures for a.) block copolymer selfassembly, b.) pattern transfer of the block copolymer template into the underlying silicon wafer substrate and grafting mono-hydroxy terminated brushes to the substrate surface, c.) reaction methods to introduce tethered ionic moieties, iv.) methods for structural and spectroscopic characterization, v.) methods for making ionic conductivity measurements on interdigitated electrodes via electrochemical impedance spectroscopy; and supplemental vi.) XPS and water contact angle data. The supporting information also provides details, additional results, and movies from atomistic MD simulations.

\section{REFERENCES}

1. Strathmann, H., Ion-Exchange Membrane Separation Processes, Volume 9. Elsevier Science: Amsterdam, The Netherlands, 2004; Vol. 9.

2. Strathmann, H.; Grabowski, A.; Eigenberger, G., Ion-Exchange Membranes in the Chemical Process Industry. Industrial \& Engineering Chemistry Research 2013, 52 (31), 10364-10379.

3. Sata, T., Ion Exchange Membranes: Preparation, Characterization, Modification and Application. Royal Society of Chemistry: Cambridge, UK, 2004.

4. Opekar, F.; Stulik, K., Electrochemical sensors with solid polymer electrolytes. Analytica Chimica Acta 1999, 385 (1-3), 151-162.

5. Palakkal, V. M.; Rubio, J.; Lin, Y. J.; Arges, C. G., Low resistant ion-exchange membranes for energy efficient membrane capacitive deionization. ACS Sustainable Chemistry \& Engineering 2018, 32 (5), 5944-5950.

6. Su, X.; Tan, K.-J.; Elbert, J.; Ruttiger, C.; Gallei, M.; Jamison, T. F.; Hatton, T. A., Asymmetric Faradaic systems for selective electrochemical separations. Energy \& Environmental Science 2017, 10 (5), 1272-1283.

7. Berson, J.; Burshtain, D.; Zeira, A.; Yoffe, A.; Maoz, R.; Sagiv, J., Single-layer ionic conduction on carboxyl-terminated silane monolayers patterned by constructive lithography. Nature Materials 2015, 14 (6), 613-621.

8. Leibler, L., Theory of microphase separation in block copolymers. Macromolecules 1980, 13 (6), 1602-17.

9. Chang, W. B.; Evans, C. M.; Popere, B. C.; Russ, B. M.; Liu, J.; Newman, J.; Segalman, R. A., Harvesting Waste Heat in Unipolar Ion Conducting Polymers. ACS Macro Letters 2016, 5 (1), 94-98. 
10. Chang, W. B.; Fang, H.; Liu, J.; Evans, C. M.; Russ, B.; Popere, B. C.; Patel, S. N.; Chabinyc, M. L.; Segalman, R. A., Electrochemical Effects in Thermoelectric Polymers. ACS Macro Letters 2016, 5 (4), 455-459.

11. Arges, C. G.; Kambe, Y.; Suh, H. S.; Ocola, L. E.; Nealey, P. F., Perpendicularly Aligned, Anion Conducting Nanochannels in Block Copolymer Electrolyte Films. Chemistry of Materials 2016, 28 (5), 1377-1389.

12. Modestino, M. A.; Paul, D. K.; Dishari, S.; Petrina, S. A.; Allen, F. I.; Hickner, M. A.; Karan, K.; Segalman, R. A.; Weber, A. Z., Self-Assembly and Transport Limitations in Confined Nafion Films. Macromolecules 2013, 46 (3), 867-873.

13. Kusoglu, A.; Kushner, D.; Paul, D. K.; Karan, K.; Hickner, M. A.; Weber, A. Z., Impact of Substrate and Processing on Confinement of Nafion Thin Films. Advanced Functional Materials 2014, 24 (30), 4763-4774.

14. Paul, D. K.; McCreery, R.; Karan, K., Proton Transport Property in Supported Nafion Nanothin Films by Electrochemical Impedance Spectroscopy. Journal of the Electrochemical Society 2014, 161 (14), F1395-F1402.

15. Arges, C. G.; Kambe, Y.; Dolejsi, M.; Wu, G.-P.; Segal-Pertz, T.; Ren, J.; Cao, C.; Craig, G. S. W.; Nealey, P. F., Interconnected ionic domains enhance conductivity in microphase separated block copolymer electrolytes. Journal of Materials Chemistry A 2017, 5 (11), 5619-5629.

16. Markovic, N.; Gasteiger, H.; Ross, P. N., Kinetics of oxygen reduction on Pt(hkl) electrodes: implications for the crystallite size effect with supported Pt electrocatalysts. Journal of the Electrochemical Society 1997, 144 (5), 1591-1597.

17. Stamenkovic, V. R.; Markovic, N. M. 2018 DOE Hydrogen and Fuel Cells Program Review: Tailored High Performance Low-PGM Alloy Cathode Catalysts; 2018.

18. Hur, S.-M.; Thapar, V.; Ramirez-Hernandez, A.; Nealey, P. F.; de Pablo, J. J., Defect Annihilation Pathways in Directed Assembly of Lamellar Block Copolymer Thin Films. ACS Nano 2018, 12 (10), 9974-9981.

19. Hur, S.-M.; Thapar, V.; Ramirez-Hernandez, A.; Khaira, G.; Segal-Peretz, T.; RinconDelgadillo, P. A.; Li, W.; Muller, M.; Nealey, P. F.; de Pablo, J. J., Molecular pathways for defect annihilation in directed self-assembly. Proceedings of the National Academy of Sciences of the United States of America 2015, 112 (46), 14144-14149.

20. Elabd, Y. A.; Walker, C. W.; Beyer, F. L., Triblock copolymer ionomer membranes. Part II. Structure characterization and its effects on transport properties and direct methanol fuel cell performance. Journal of Membrane Science 2004, 231 (1-2), 181-188.

21. Arges, C. G.; Parrondo, J.; Johnson, G.; Nadhan, A.; Ramani, V., Assessing the influence of different cation chemistries on ionic conductivity and alkaline stability of anion exchange membranes. Journal of Materials Chemistry 2012, 22 (9), 3733-3744.

22. Kambe, Y.; Arges, C. G.; Patel, S. N.; Stoykovich, M. P.; Nealey, P. F., Ion Conduction in Microphase-Separated Block Copolymer Electrolytes. The Electrochemical Society Interface 2017, 26 (1), 61-67.

23. Elabd, Y. A.; Hickner, M. A., Block Copolymers for Fuel Cells. Macromolecules 2011, 44 (1), 1-11.

24. Li, N.; Guiver, M. D., Ion Transport by Nanochannels in Ion-Containing Aromatic Copolymers. Macromolecules 2014, 47 (7), 2175-2198.

25. Young, W.-S.; Kuan, W.-F.; Epps, T. H., III, Block copolymer electrolytes for rechargeable lithium batteries. Journal of Polymer Science, Part B: Polymer Physics 2014, 52 (1), 1-16. 
26. Hoarfrost, M. L.; Segalman, R. A., Ionic Conductivity of Nanostructured Block Copolymer/Ionic Liquid Membranes. Macromolecules 2011, 44 (13), 5281-5288.

27. Hoarfrost, M. L.; Segalman, R. A., Conductivity Scaling Relationships for Nanostructured Block Copolymer/Ionic Liquid Membranes. ACS Macro Letters 2012, 1 (8), 937-943.

28. Park, M. J.; Balsara, N. P., Phase Behavior of Symmetric Sulfonated Block Copolymers. Macromolecules 2008, 41 (10), 3678-3687.

29. Park, M. J.; Balsara, N. P., Anisotropic Proton Conduction in Aligned Block Copolymer Electrolyte Membranes at Equilibrium with Humid Air. Macromolecules 2010, 43 (1), 292298.

30. Chintapalli, M.; Chen, X. C.; Thelen, J. L.; Teran, A. A.; Wang, X.; Garetz, B. A.; Balsara, N. P., Effect of Grain Size on the Ionic Conductivity of a Block Copolymer Electrolyte. Macromolecules 2014, 47 (15), 5424-5431.

31. Inceoglu, S.; Rojas, A. A.; Devaux, D.; Chen, X. C.; Stone, G. M.; Balsara, N. P., Morphology-Conductivity Relationship of Single-Ion-Conducting Block Copolymer Electrolytes for Lithium Batteries. ACS Macro Letters 2014, 3 (6), 510-514.

32. Rojas, A. A.; Inceoglu, S.; Mackay, N. G.; Thelen, J. L.; Devaux, D.; Stone, G. M.; Balsara, N. P., Effect of Lithium-Ion Concentration on Morphology and Ion Transport in Single-Ion-Conducting Block Copolymer Electrolytes. Macromolecules 2015, 48 (18), 6589-6595.

33. Villaluenga, I.; Chen, X. C.; Devaux, D.; Hallinan, D. T.; Balsara, N. P., NanoparticleDriven Assembly of Highly Conducting Hybrid Block Copolymer Electrolytes. Macromolecules 2015, 48 (2), 358-364.

34. Majewski, P. W.; Gopinadhan, M.; Jang, W.-S.; Lutkenhaus, J. L.; Osuji, C. O., Anisotropic Ionic Conductivity in Block Copolymer Membranes by Magnetic Field Alignment. Journal of the American Chemical Society 2010, 132 (49), 17516-17522.

35. Majewski, P. W.; Gopinadhan, M.; Osuji, C. O., Understanding anisotropic transport in self-assembled membranes and maximizing ionic conductivity by microstructure alignment. Soft Matter 2013, 9 (29), 7106-7116.

36. Feng, X.; Tousley, M. E.; Cowan, M. G.; Wiesenauer, B. R.; Nejati, S.; Choo, Y.; Noble, R. D.; Elimelech, M.; Gin, D. L.; Osuji, C. O., Scalable Fabrication of Polymer Membranes with Vertically Aligned $1 \mathrm{~nm}$ Pores by Magnetic Field Directed Self-Assembly. ACS Nano 2014, 8 (12), 11977-11986.

37. Park, M. J.; Downing, K. H.; Jackson, A.; Gomez, E. D.; Minor, A. M.; Cookson, D.; Weber, A. Z.; Balsara, N. P., Increased water retention in polymer electrolyte membranes at elevated temperatures assisted by capillary condensation. Nano Letters 2007, 7 (11), 35473552.

38. Stoykovich, M. P.; Nealey, P. F., Block copolymers and conventional lithography. Materials Today 2006, 9 (9), 20-29.

39. Ji, S.; Wan, L.; Liu, C.-C.; Nealey, P. F., Directed self-assembly of block copolymers on chemical patterns: A platform for nanofabrication. Progress in Polymer Science 2016, 5455, 76-127.

40. Cummins, C.; Ghoshal, T.; Holmes, J. D.; Morris, M. A., Strategies for Inorganic Incorporation using Neat Block Copolymer Thin Films for Etch Mask Function and Nanotechnological Application. Advanced Materials 2016, 28 (27), 5586-5618.

41. Beers, K. M.; Hallinan, D. T.; Wang, X.; Pople, J. A.; Balsara, N. P., Counterion Condensation in Nafion. Macromolecules 2011, 44 (22), 8866-8870. 
42. Beers, K. M.; Balsara, N. P., Design of Cluster-free Polymer Electrolyte Membranes and Implications on Proton Conductivity. ACS Macro Letters 2012, 1 (10), 1155-1160.

43. Kamcev, J.; Paul, D. R.; Freeman, B. D., Ion Activity Coefficients in Ion Exchange Polymers: Applicability of Manning's Counterion Condensation Theory. Macromolecules 2015, 48 (21), 8011-8024.

44. Kamcev, J.; Galizia, M.; Benedetti, F. M.; Jang, E.-S.; Paul, D. R.; Freeman, B. D.; Manning, G. S., Partitioning of mobile ions between ion exchange polymers and aqueous salt solutions: importance of counter-ion condensation. Physical Chemistry Chemical Physics 2016, 18 (8), 6021-6031.

45. Kamcev, J.; Paul, D. R.; Manning, G. S.; Freeman, B. D., Accounting for frame of reference and thermodynamic non-idealities when calculating salt diffusion coefficients in ion exchange membranes. Journal of Membrane Science 2017, 537, 396-406.

46. Kamcev, J.; Paul, D. R.; Manning, G. S.; Freeman, B. D., Predicting Salt Permeability Coefficients in Highly Swollen, Highly Charged Ion Exchange Membranes. ACS Applied Materials \& Interfaces 2017, 9 (4), 4044-4056.

47. Kamcev, J.; Doherty, C. M.; Lopez, K. P.; Hill, A. J.; Paul, D. R.; Freeman, B. D., Effect of fixed charge group concentration on salt permeability and diffusion coefficients in ion exchange membranes. Journal of Membrane Science 2018, 566, 307-316.

48. Kamcev, J.; Paul, D. R.; Manning, G. S.; Freeman, B. D., Ion Diffusion Coefficients in Ion Exchange Membranes: Significance of Counterion Condensation. Macromolecules 2018, 51 (15), 5519-5529.

49. Kelley, T. W.; Schorr, P. A.; Johnson, K. D.; Tirrell, M.; Frisbie, C. D., Direct Force Measurements at Polymer Brush Surfaces by Atomic Force Microscopy. Macromolecules 1998, 31 (13), 4297-4300.

50. Balastre, M.; Li, F.; Schorr, P.; Yang, J.; Mays, J. W.; Tirrell, M. V., A Study of Polyelectrolyte Brushes Formed from Adsorption of Amphiphilic Diblock Copolymers Using the Surface Forces Apparatus. Macromolecules 2002, 35 (25), 9480-9486.

51. Stoykovich, M. P.; Kang, H.; Daoulas, K. C.; Liu, G.; Liu, C.-C.; de Pablo, J. J.; Mueller, M.; Nealey, P. F., Directed Self-Assembly of Block Copolymers for Nanolithography: Fabrication of Isolated Features and Essential Integrated Circuit Geometries. ACS Nano 2007, 1 (3), 168-175.

52. Han, E.; Stuen, K. O.; La, Y.-H.; Nealey, P. F.; Gopalan, P., Effect of Composition of Substrate-Modifying Random Copolymers on the Orientation of Symmetric and Asymmetric Diblock Copolymer Domains. Macromolecules 2008, 41 (23), 9090-9097.

53. Simms, J. A., Epoxide-substituted vinyl and acrylate copolymers. Journal of Applied Polymer Science 1961, 5 (13), 58-63.

54. Muzammil, E. M.; Khan, A.; Stuparu, M. C., Post-polymerization modification reactions of poly(glycidyl methacrylate)s. RSC Advances 2017, 7 (88), 55874-55884.

55. Bennett, T.; Pei, K.; Cheng, H. H.; Thurecht, K. J.; Jack, K. S.; Blakey, I., Extending the scope of poly(styrene)-block-poly(methyl methacrylate) for directed self assembly. Proceedings of SPIE 2014, 9049, 90490R/1-90490R/12.

56. Diederichsen, K. M.; Brow, R. R.; Stoykovich, M. P., Percolating Transport and the Conductive Scaling Relationship in Lamellar Block Copolymers under Confinement. ACS Nano 2015, 9 (3), 2465-2476. 
57. Chen, X. C.; Wong, D. T.; Yakovlev, S.; Beers, K. M.; Downing, K. H.; Balsara, N. P., Effect of morphology of nanoscale hydrated channels on proton conductivity in block copolymer electrolyte membranes. Nano Letters 2014, 14 (7), 4058-64.

58. Martins, C. R.; Ruggeri, G.; De Paoli, M.-A., Synthesis in pilot plant scale and physical properties of sulfonated polystyrene. Journal of the Brazilian Chemical Society 2003, 14 (5), 797-802.

59. Olmos, D.; Martin, E. V.; Gonzalez-Benito, J., New molecular-scale information on polystyrene dynamics in PS and PS-BaTiO3 composites from FTIR spectroscopy. Physical Chemistry Chemical Physics 2014, 16 (44), 24339-24349.

60. Dean, J. A., Analytical Chemistry Handbook. McGraw-Hill, Inc.: New York, 1995; p 6.28.

61. Olvera de la Cruz, M.; Belloni, L.; Delsanti, M.; Dalbiez, J. P.; Spalla, O.; Drifford, M., Precipitation of highly charged polyelectrolyte solutions in the presence of multivalent salts. Journal of Chemical Physics 1995, 103 (13), 5781-91.

62. Brettmann, B. K.; Laugel, N.; Hoffmann, N.; Pincus, P.; Tirrell, M., Bridging contributions to polyelectrolyte brush collapse in multivalent salt solutions. Journal of Polymer Science, Part A: Polymer Chemistry 2016, 54 (2), 284-291.

63. Jackson, N. E.; Brettmann, B. K.; Vishwanath, V.; Tirrell, M.; de Pablo, J. J., Comparing Solvophobic and Multivalent Induced Collapse in Polyelectrolyte Brushes. ACS Macro Letters 2017, 6 (2), 155-160.

64. Zhulina, E. B.; Wolterink, J. K.; Borisov, O. V., Screening Effects in a Polyelectrolyte Brush: Self-Consistent-Field Theory. Macromolecules 2000, 33 (13), 4945-4953.

65. Mei, Y.; Lauterbach, K.; Hoffmann, M.; Borisov, O. V.; Ballauff, M.; Jusufi, A., Collapse of Spherical Polyelectrolyte Brushes in the Presence of Multivalent Counterions. Physical Review Letters 2006, 97 (15), 158301/1-158301/4.

66. Ahrens, H.; Forster, S.; Helm, C. A., Charged Polymer Brushes: Counterion Incorporation and Scaling Relations. Physical Review Letters 1998, 81 (19), 4172-4175.

67. Manning, G. S., Limiting laws and counterion condensation in polyelectroyte solutions. I. Colligative properties. Journal of Chemical Physics 1969, 51 (3), 924-33.

68. Jorgensen, W. L.; Maxwell, D. S.; Tirado-Rives, J., Development and Testing of the OPLS All-Atom Force Field on Conformational Energetics and Properties of Organic Liquids. Journal of the American Chemical Society 1996, 118 (45), 11225-11236.

69. Wu, Y.; Chen, H.; Wang, F.; Paesani, F.; Voth, G. A., An Improved Multistate Empirical Valence Bond Model for Aqueous Proton Solvation and Transport. Journal of Physical Chemistry B 2008, 112 (2), 467-482. 
Table of Contents Artwork
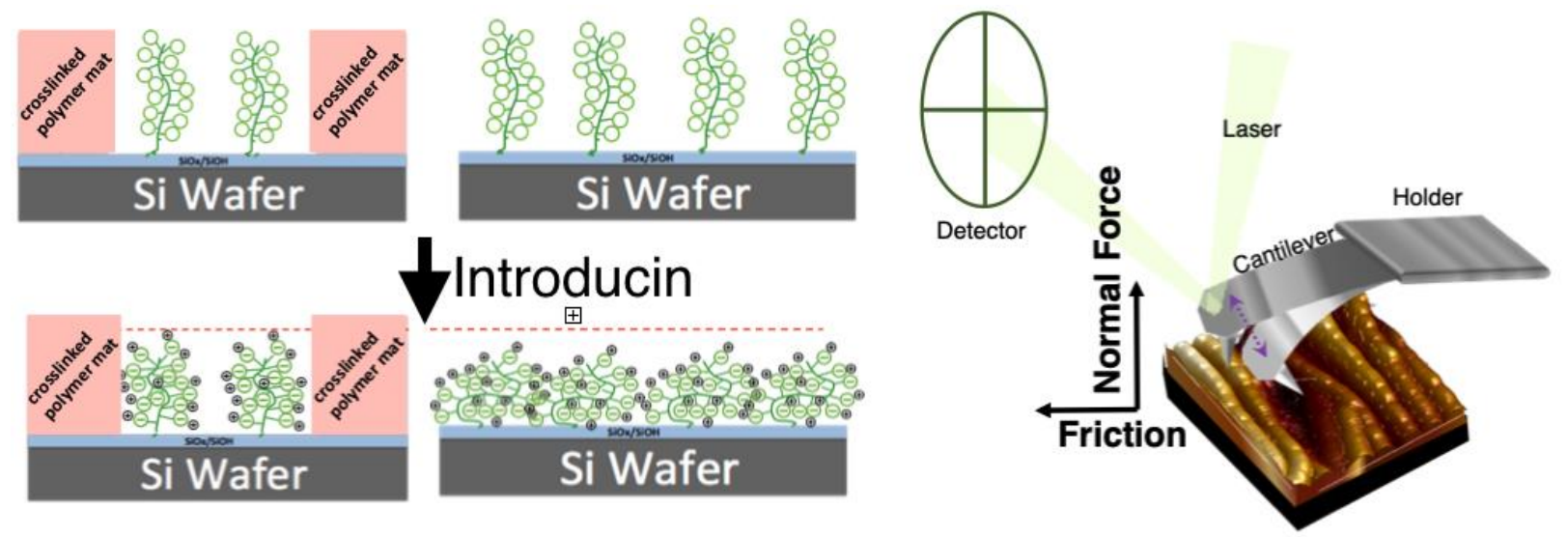